\title{
Theoretical Assessment of a Repolarization Time Marker Based on the Intracardiac Bipolar Electrogram
}

\author{
Michele Orini ${ }^{1,2}$, Stefan Van Duijvenboden ${ }^{1}$, Neil Srinivasan ${ }^{2}$, Malcolm Finlay ${ }^{2}$, \\ Peter Taggart $^{1}$, Pier D Lambiase ${ }^{1,2}$ \\ ${ }^{1}$ University College London, London, United Kingdom \\ ${ }^{2}$ Barts Heart Centre, St Bartholomew's Hospital, London, United Kingdom
}

\begin{abstract}
The spatio-temporal organization of cardiac repolarization modulates the vulnerability to dangerous ventricular arrhythmias. Methodologies that provide accurate assessment of cardiac repolarization are of primary importance for a better understanding of cardiac electrophysiology and represent a potentially useful tool for clinical applications. The most commonly used repolarization time (RT) marker from extracellular recordings is derived from the unipolar electrogram (UEG). However, far field potentials and remote activity may in certain conditions bias this marker. In this paper, a RT marker based on the bipolar electrogram (BEG) is proposed. An analytical expression of the BEG based on a simple model of the cardiac extracellular potential is derived. According to the proposed analytical framework the BEG exhibits a repolarization wave whose extremum (maximum or minimum) corresponds to the average of the local RTs at the two electrodes of the bipole. The amplitude of this extremum is a function of the steepness of phase 3 of the action potentials, inter-electrode distance, conduction velocity and direction of wave-back propagation. A simulation study based on this analytical framework showed that for noisy to good signal quality (SNR of the $U E G \geq 10 \mathrm{~dB}$ ), and for a typical inter-electrode distance of $2 \mathrm{~mm}$, conduction velocity between 0.2 and $0.6 \mathrm{~m} / \mathrm{s}$, and an angle between conduction direction and the inter-electrode axis $\leq \pi / 4$, the median absolute error was lower than $6.8 \mathrm{~ms}$ while the median linear correlation between estimated and theoretical RT was higher than 0.91. Examples of RT derived from $B E G$ recorded in a structurally normal heart in both the right and left ventricles demonstrate that the proposed procedure is feasible in human in-vivo studies.
\end{abstract}

\section{Introduction}

The spatio-temporal organization of cardiac repolarization modulates the vulnerability to dangerous ventricular arrhythmia and sudden cardiac death [1]. Methodologies that provide accurate assessment of cardiac repolarization are of primary importance for a better understanding of cardiac electrophysiology and represent a potentially useful tool for clinical applications. The most commonly used repolarization time (RT) marker from extracellular recordings is the time of the maximum upslope of the T-wave in the unipolar electrogram (UEG). Previous theoretical [2-5] and experimental [6] work has demonstrated that the RT markers from the intracardiac UEG is accurate in ideal conditions, but its accuracy critically depends on remote components (far fields, distant activity etc.) that affect the T-wave of the UEG. Extracellular potentials recorded in a bipolar configuration as the difference between UEG from two adjacent cardiac sites, so called bipolar electrograms (BEG), are commonly used in electrophysiological studies to measure local activation time, but not RT. The bipolar configuration has the advantage of offering information that only depends on local repolarization dynamics and is (almost) independent of remote electrical activity, which being (almost) equal at both electrodes of the bipole is canceled. The purpose of this paper is to conduct a theoretical investigation of a cardiac RT marker based on the BEG along with supportive human data.

\section{Method}

\subsection{Conceptual Framework}

Simulated transmebrane and extracellular potentials with realistic shapes are generated using a simple analytical model as in previous studies $[7,8]$. The repolarization phase of an action potential (AP) recorded at a given cardiac site $x_{i}$ is defined by a logistic function (Fig. 1):

$V_{i}(t)=V\left(t, x_{i}\right)=A\left(1-\frac{1}{1+\exp \left(-\beta\left(t-\tau_{i}\right)\right)}\right)-V_{0}$

where $\beta$ is a constant that determines the steepness of the repolarization downslope during phase 3 of all APs, 
$A$ and $V_{0}$ are the amplitude and the resting potential of $V_{i}(t) . \tau_{i}$ is the RT at cardiac site $x_{i}$, defined as the the moment of the steepest downslope in the AP, i.e. $\tau_{i}=$ $\arg \min \left(d V_{i}(t) / d t\right)$. According to a simple model, the UEG recorded at the same cardiac site is [4]:

$$
U\left(t, x_{i}\right)=C\left(V_{\mathrm{R}}(t)-V_{i}(t)\right)
$$

where $C$ is a scaling factor that takes into account the difference between intra- and extracellular conductances [4], and $V_{\mathrm{R}}(t)$ is a position-independent potential that represents far field activities, defined as the arithmetic mean of all $V\left(t, x_{i}\right)$. A bipolar electrogram, $B_{i, j}(t)$, is defined as the potential difference between two adjacent extracellular cardiac sites $x_{i}$ and $x_{j}$ (Fig. 1):

$$
B_{i, j}(t)=U\left(t, x_{j}\right)-U\left(t, x_{i}\right)=C\left(V_{i}(t)-V_{j}(t)\right)
$$

Inserting (1) into (3), the analytical expression of the bipolar electrogram becomes:

$$
B_{i, j}(t)=A C \frac{\exp \left(k_{i}\right)-\exp \left(k_{j}\right)}{\left(\exp \left(k_{i}\right)+1\right)\left(\exp \left(k_{j}\right)+1\right)}
$$

where

$$
k_{i}=-\beta\left(t-\tau_{i}\right)=-\beta\left(t-\tau_{0}+\Delta \tau / 2\right)
$$

$\tau_{0}=\left(\tau_{i}+\tau_{j}\right) / 2$ and $\Delta \tau=\tau_{i}-\tau_{j}$. In $2 \mathrm{D}$, the interelectrode repolarization interval is a function of the interelectrode distance, $d$, conduction (wave-back) velocity, $v$, and direction, $\theta$, i.e. $\Delta \tau=(d \cos \theta) / v$. It is worth noting that in this framework, UEG and RT are both affected by the remote component $V_{\mathrm{R}}(t)$, while the BEG only depends on local transmembrane potentials. Also, the BEG as defined in (3) has a local extremum (either a maximum or a minimum) in correspondence of $\tau_{0}$.

In real signals contaminated by noise, the likelihood of correctly localize the extremum of the BEG corresponding to $\tau_{0}$ is determined by its amplitude. The amplitude can be numerically obtained from (4). However, an analytical approximation is obtained by considering $\exp \left(k_{i}\right) \approx k_{i}+1$ for $t \rightarrow \tau_{i}$ and $\Delta \tau \rightarrow 0$. Then, the amplitude of the extremum associated with local RT is:

$$
B_{i, j}\left(t=\tau_{0}\right) \approx \frac{4 \beta A C \Delta \tau}{16-\beta^{2} \Delta \tau^{2}} \approx-\frac{A C \beta d \cos \theta}{4 v}
$$

This expression shows that the repolarization wave of the BEG increases with larger and steeper AP (through $A$ and $\beta$ ) and larger inter-electrode distance, $d$, while it decreases for increasing conduction velocity, $v$, and for $\theta$ approaching $90^{\circ}$. Figure 2 shows the amplitude of the repolarization wave, $B_{i, j}\left(t=\tau_{0}\right)$, as a function of $\{\beta, d, v, \theta\}$.
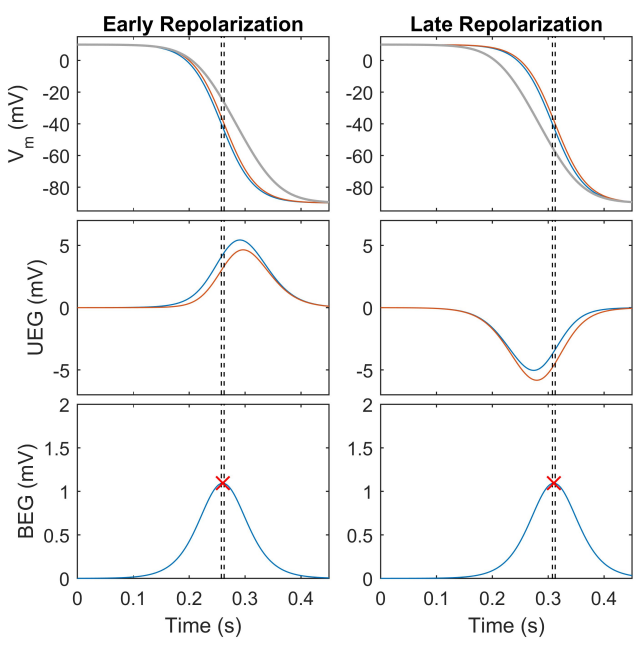

Figure 1. From top to bottom: Action potentials and the remote component (bold grey line), UEGs exhibiting positive (left) and negative (right) T-waves (associated with early and late RT, respectively) and BEG. Vertical lines represent RT $\tau_{i}$ and $\tau_{j}$. The extremum of the BEG corresponds to $\tau_{0}=\left(\tau_{i}+\tau_{j}\right) / 2$.

\subsection{Simulation Study}

As shown in Fig. 3, a squared grid of $5 \times 5 \mathrm{~cm}$ was considered. Four lines of 10 electrodes were distributed to simulate 4 decapolar catheters. Parameters were $d=\{1,2,4\} \mathrm{mm}, v=\{0.2,0.4,0.6\} \mathrm{m} / \mathrm{s}, \theta=$ $\{0, \pi / 8, \pi / 4,3 / 8 \pi\}, \beta=35 \mathrm{~s}^{-1}$, and sampling frequency was $4 \mathrm{KHz}$. RT at each point $x_{i}=(a, b)$ was defined as:

$$
\tau_{x_{i}}=\tau_{i}=\frac{a \cos \theta+b \sin \theta}{v}+\tau_{\min }
$$

with $\tau_{\min }=225 \mathrm{~ms}$. Trasmembrane potentials were generated using (1). White Guassian noise was created for each point of the grid and spatially filtered with a disk of radius equal to $5 \mathrm{~mm}$ in order to introduce a spatial correlation between noise contamination in adjacent electrodes as observed experimentally. Noise with $\mathrm{SNR}=\{5,10,15,20\}$ $\mathrm{dB}$ was added to $U_{i}(t)$, and $B_{i, j}(t)$ was computed as in (3). Bipolar electrograms were low pass filtered at $25 \mathrm{~Hz}$ and RT, $\hat{\tau}_{i, j}$, were estimated as the time of the extremum in the BEG. For each configuration $\{v, d, \theta, \mathrm{SNR}\}$, the simulation process was repeated 25 times providing 1000 RT estimates. The absolute error $\left|\hat{\tau}_{i, j}-\tau_{i}\right|$ and the correlation coefficient between $\hat{\tau}_{i, j}$ and $\tau_{i}$ were computed.

\subsection{Experimental Data}

During an electrophysiological study for supraventricular tachycardia in a patient with structurally normal ventricles, two decapolar catheters were placed in the left and 
right ventricles. Unipolar electrograms were referenced against the WCT, sampled at $2 \mathrm{KHz}$ and recorded with amplifier with bandwidth $0.05-500 \mathrm{~Hz}$ as in previous studies [9]. Ventricular pacing was established from the RV apex. Bipolar electrograms were computed off-line as the difference between UEG from 5 pairs of adjacent poles $(d=2$ $\mathrm{mm})$. Bespoke algorithms were used for the analysis as in previous studies $[10,11]$.

\section{Results}

For levels of noise that corresponded to UEG with $\mathrm{SNR} \geq 15 \mathrm{~dB}$, the median absolute error was lower than $10 \mathrm{~ms}$ while the median correlation coefficient was higher than 0.90 , for all combinations of $\{d, v, \theta\}$ (Fig. 4). These levels of noise are considered normal in human recordings from cathlabs and cardiac theaters [12]. In these conditions, $\hat{\tau}_{i, j} \approx \tau_{0}$, and parameters associated with smaller $\Delta \tau$, i.e. lower $d$ and higher $v$, provided better RT estimates because $\left|\tau_{i}-\tau_{0}\right|=\left|\tau_{j}-\tau_{0}\right|=\Delta \tau / 2$. However, for $\mathrm{SNR} \leq 10 \mathrm{~dB}$, combinations of parameters associated with longer $\Delta \tau$ may provide better estimates because a long $\Delta \tau$ is associated with a higher amplitude of the repolarization wave in the BEG. In general, for noisy to good quality signals (SNR of the UEG $\geq 10 \mathrm{~dB}$ ) and for a typical inter-electrode distance $d=2 \mathrm{~mm}$, a conduction velocity $0.2 \leq v \leq 0.6 \mathrm{~m} / \mathrm{s}$, and an angle between conduction direction and the inter-electrode axis $\theta \leq \pi / 4$, the median absolute error was lower than $6.8 \mathrm{~ms}$ and the median linear correlation with theoretical values was higher than 0.91 .

Figure 5 shows the repolarization wave of UEG and BEG from a human heart recorded in the RV and LV endocardium during ventricular pacing. The BEG exhibited a repolarization wave whose extremum $\tau_{0}$ (vertical line) corresponded to the upslope of both the positive and negative UEG T-wave. The amplitude of the BEG repolarization wave was about $15 \%$ of the UEG T-wave.

\section{Discussion}

This paper provides the first theoretical investigation of a RT marker based on the BEG, a signal that has the advantage of being the sole results of local repolarization dynamics and largely independent of far field potentials and remote activities. This marker has the potential to overcome the limitations of the traditional markers based on the UEG $[2,3,6,13]$. According to the simple analytical framework used in this study, the extremum of the BEG corresponds to the mean of the RT at the two electrodes of the bipole. The amplitude of this extremum is a function of the amplitude and shape of the local transmembrane potentials, the relation between intra- and extra-cellular conductance and the inter-electrode repolarization interval, which in turn depends on the inter-electrode distance, the local conduc-
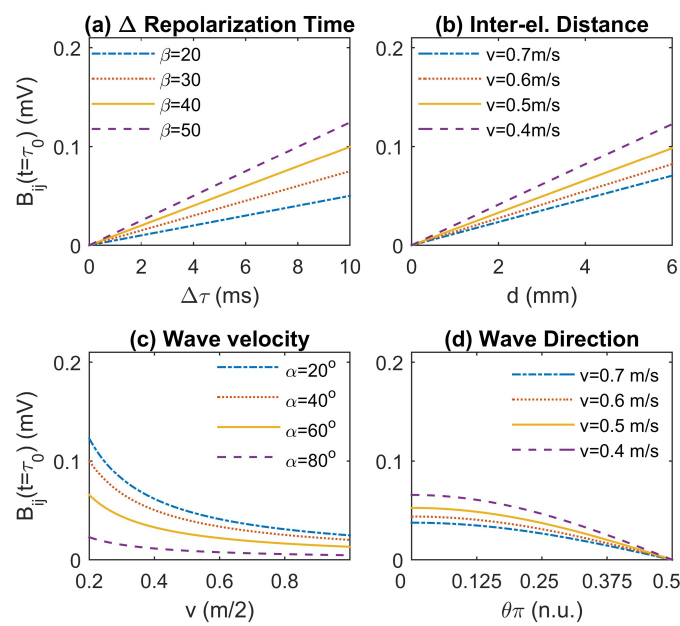

Figure 2. Amplitude of the repolarization wave in the bipolar electrogram as a function of inter-electrode repolarization interval $\Delta \tau$, inter-electrode distance, $d$, conduction velocity, $v$, and conduction direction, $\theta$. These curves are computed using (4), for $t=\tau_{0}$ and $A C=1$.

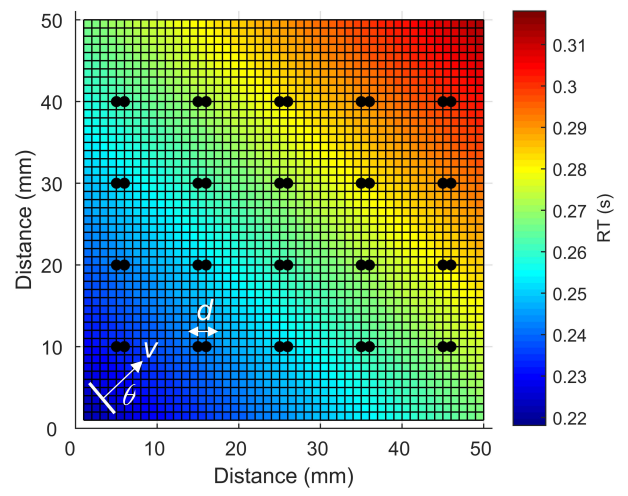

Figure 3. Schematic of the simulation study, representing electrodes from 4 decapolar catheters (black dots). Parameters $d, v$ and $\theta$ represent the inter-electrode distance, conduction velocity and direction, respectively. Colors represent a uniform repolarization wave as in (7).
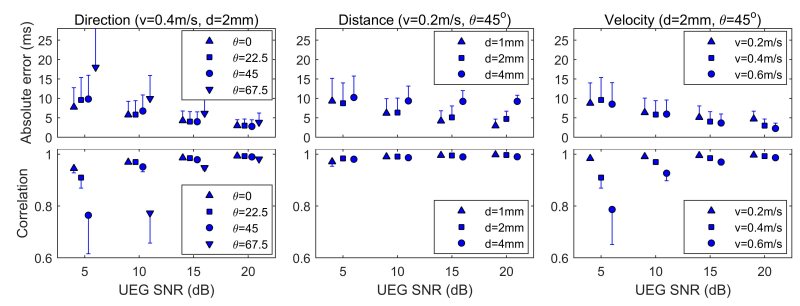

Figure 4. Results of the 2D simulation study. Upper and lower panels represent absolute error and linear correlation coefficient (median \pm median absolute deviation) as a function of SNR added to the UEG, inter-electrode distance, $d$, conduction velocity, $v$, and direction, $\theta$. 

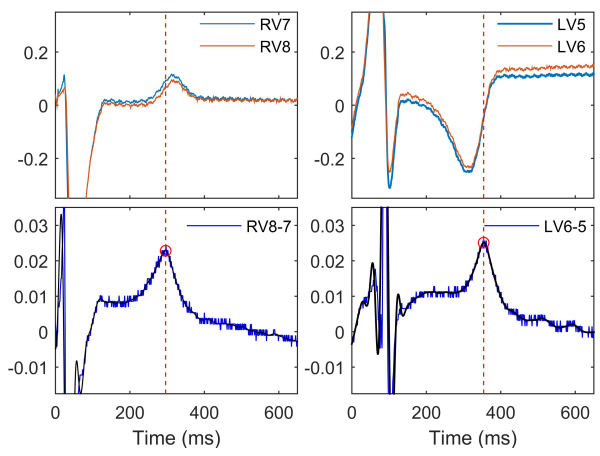

Figure 5. Unipolar (top) and bipolar (bottom) recordings from an intact human heart recorded in the right (left panel) and left (right panel) ventricle during ventricular pacing. The BEG were computed as the difference of the UEG. In the bottom panel, blue and black lines represent unfiltered and filtered signals, respectively. Vertical line represents RT marker from the BEG.

tion velocity and direction. An analytical expression that describes these relations has been proposed. The simulation study shows that although the RT measured from the BEG can be accurate even in noisy signals, it critically depends on parameters such as conduction velocity and direction. The analysis of recordings from an intact human heart suggests that this marker may be applied to the study of the spatio-temporal organization of human cardiac repolarization, with potentially interesting applications in cardiac mapping.

The analytical expression implemented to simulate transembrane potentials was based on [14], while the description of the UEG in terms of local and remote components was based on [4]. The proposed model assumes isotropic conditions, uniform and planar wavefronts for electrical recovery, and describes phase 3 of the cardiac action potential as a logistic function. Its main purpose is to provide a simple conceptual framework for the study of the BEG, rather than to reproduce realistic conditions.

\section{References}

[1] Ramírez J, Orini M, Mincholé A, Monasterio V, Cygankiewicz I, de Luna AB, Martínez JP, Pueyo E, Laguna P. T-wave morphology restitution predicts sudden cardiac death in patients with chronic heart failure. J Am Heart Assoc 2017;6(5):e005310. ISSN 20479980.

[2] Steinhaus BM. Estimating cardiac transmembrane activation and recovery times from unipolar and bipolar extracellular electrograms: a simulation study. Circ Res 1989; 64(3):449-462.

[3] Scacchi S, Franzone PC, Pavarino LF, Taccardi B. A reliability analysis of cardiac repolarization time markers. Mathematical Biosciences 2009;219(2):113-128.

[4] Potse M, Vinet A, Opthof T, Coronel R. Validation of a simple model for the morphology of the $\mathrm{T}$ wave in unipolar electrograms. Am J Physiol Heart Circ Physiol 2009; 297(2):H792-H801.

[5] Van Duijvenboden S, Orini M, Taggart P, Hanson B. Accuracy of measurements derived from intracardiac unipolar electrograms: A simulation study. In Conf Proc IEEE Eng Med Biol Soc, volume 2015. 2015; 76-79.

[6] Coronel R, de Bakker JMT, Wilms-Schopman FJG, Opthof T, Linnenbank AC, Belterman CN, Janse MJ. Monophasic action potentials and activation recovery intervals as measures of ventricular action potential duration: Experimental evidence to resolve some controversies. Heart Rhythm 2006;3(9):1043-1050.

[7] Orini M, Hanson B, Taggart P, Lambiase P. Detection of transient, regional cardiac repolarization alternans by timefrequency analysis of synthetic electrograms. Conf Proc IEEE Eng Med Biol Soc 2013;3773-3776.

[8] Orini M, Taggart P, Hayward M, Lambiase P. On how 2:1 conduction block can induce t-wave alternans in the unipolar intracavitary electrogram: Modelling in-vivo human recordings from an ischemic heart. Conf Proc IEEE Eng Med Biol Soc Aug 2015;2015:5676-5679.

[9] Srinivasan NT, Orini M, Simon RB, Providência R, Khan F, Segal OR, Babu G, Bradley R, Rowland E, Ahsan S, Chow AW, Lowe M, Taggart P, Lambiase P. Ventricular Stimulus Site Influences Dynamic Dispersion of Repolarization In The Intact Human Heart. Am J Physiol Heart Circ Physiol jul 2016;ajpheart.00159.2016.

[10] Orini M, Hanson B, Monasterio V, Martínez JP, Hayward M, Taggart P, Lambiase P. Comparative evaluation of methodologies for T-wave alternans mapping in electrograms. IEEE Trans Biomed Eng 2014;61(2).

[11] Orini M, Taggart P, Srinivasan N, Hayward M, Lambiase PD. Interactions between activation and repolarization restitution properties in the intact human heart: In-vivo whole-heart data and mathematical description. PLoS ONE 2016;11(9):e0161765.

[12] Taggart P, Orini M, Hanson B, Hayward M, Clayton R, Dobrzynski H, Yanni J, Boyett M, Lambiase PDP. Developing a novel comprehensive framework for the investigation of cellular and whole heart electrophysiology in the in situ human heart: Historical perspectives, current progress and future prospects. Prog Biophys Mol Biol 2014;115(2-3):252260.

[13] Western D, Hanson B, Taggart P. Measurement bias in activation-recovery intervals from unipolar electrograms. Am J Physiol Heart Circ Physiol 2015;308(4):H331-H338.

[14] Van Dam PM, Oostendorp TF, Linnenbank AC, Van Oosterom A. Non-invasive imaging of cardiac activation and recovery. Annals of Biomedical Engineering 2009; 37(9):1739-1756.

Address for correspondence:

Name: Michele Orini

University College London, Gower Street, London, UK.

E-mail address: m.orini@ucl.ac.uk 